\title{
Análisis de la relación confianza-compromiso en la banca en internet
}

\author{
Torres, Eduardo* \\ Manzur, Enrique** \\ Olavarrieta, Sergio*** \\ Barra, Cristóbal ${ }^{\star * * *}$
}

\section{Resumen}

El propósito de este artículo es analizar empíricamente la relación confianza-compromiso en el contexto de la banca en internet. Para lograr este objetivo, se aplicó una encuesta telefónica a un grupo de clientes de la banca en internet en Chile y se analizó detalladamente la estructura dimensional de la confianza. Por medio de un modelo de ecuaciones estructurales se pudo comprobar el efecto positivo y directo que tiene la confianza sobre el compromiso en el contexto de estudio. Además, se pudo determinar que la confianza en la banca en Internet tiene una estructura multidimensional constituida por la honestidad, benevolencia y competencia. Estos resultados otorgan a los bancos un conocimiento respecto a cuáles son los factores que deben gestionar para lograr el compromiso de los clientes que realizan transacciones a través de internet, principalmente en un contexto latinoamericano.

Palabras clave: Confianza, compromiso, banca en internet, ecuaciones estructurales.

* Doctor en Gestión, Universidad de Zaragoza, España. Profesor Asistente, Facultad de Economía y Negocios, Universidad de Chile, Santiago, Chile. eduardot@fen.uchile.cl (Autor para la Correspondencia).

** PhD in Business Administration, University of Georgia, USA. Profesor Asistente, Facultad de Economía y Negocios, Universidad de Chile, Santiago, Chile. emanzur@fen.uchile.cl

*** PhD in Business Administration, University of Georgia, USA. Profesor Asociado, Facultad de Economía y Negocios, Universidad de Chile. sergio.olavarrieta@udp.cl

Magister en Marketing, Universidad de Chile, Chile. Académico Ayudante, Facultad de Economía y Negocios, Universidad de Chile, Santiago, Chile.cbarra@fen.uchile.cl 


\title{
Analysis of the Trust-Commitment Relationship in Internet Banking
}

\begin{abstract}
The purpose of this article is to empirically analyze the trust-commitment relationship in the context of internet banking. To achieve this objective, a telephone survey was applied to a group of internet banking clients in Chile, and the dimensional structure of trust was deeply analyzed. Through a structural equation model, the positive and direct effect of trust on commitment was proven in the context of the study. Furthermore, it could be determined that trust in internet banking has a multidimensional structure constituted by honesty, benevolence and competence. These results give the banks knowledge about what factors must be managed in order to achieve commitment from the customers who make internet transactions, mainly in a Latin American context.
\end{abstract}

Key words: Trust, commitment, internet banking, structural equations.

\section{Introducción}

Tanto en el contexto tradicional como en internet, el compromiso se manifiesta en el cliente como una intención voluntaria de mantener una relación que valora (Moorman et al., 1992; Cater y Zabkar, 2008). Desde esta perspectiva, el compromiso va acompañado de una actitud positiva (Liu et al., 2006); que tiene como efecto, una resistencia al cambio (Young y Denize, 1995; N'Goala, 2006), una mayor repetición de compra y una posterior lealtad del consumidor ( $\mathrm{Ca}$ ter y Zabkar, 2008).

En el caso particular de las relaciones online, el compromiso cumple un papel especialmente relevante. De hecho, genera la intención de seguir utilizando un determinado sitio web en el futuro, motiva su recomendación, alienta su defensa ante las críticas, vuelve a los clientes más insensibles ante pequeñas fallas del sistema y también más inmunes a las ofertas de otros sitios web (Casalo et al., 2007).

Otro factor que está relacionado con el compromiso y que también es rele- vante en este contexto, es la confianza. De hecho, se ha podido constatar, que la confianza es un factor clave en el proceso de adopción de la banca en internet (Rexha et al., 2003), ya que disminuye la incertidumbre de los clientes, en relación a como el banco cumple con la entrega del servicio y responde ante posibles fallas en el sistema.

Esta situación, no siempre permite a los bancos en internet crear de manera fluida vínculos más estrechos, que se podrían traducir en relaciones de compromiso por parte de los clientes.

$\mathrm{Si}$ se considera que la confianza cumple un importante papel como factor regulador de este riesgo percibido (Garbarino y Johnson, 1999), la tarea fundamental de los bancos en internet, debería centrarse en gestionar la confianza para así alcanzar el compromiso de sus clientes. Es decir, gestionar eficientemente cada uno de los componentes de la confianza para así lograr que los clientes hagan verdaderos esfuerzos por mantener una relación duradera con su banco a través de internet. 
De acuerdo a lo anterior, y considerando que en este contexto bancario, y especialmente en Latinoamérica existen pocos análisis empíricos sobre esta materia, el presente estudio se plantea como objetivo, contrastar si en el contexto de la banca en internet la confianza es un factor relevante para lograr el compromiso de sus clientes. Para lograr este objetivo, se analiza la estructura dimensional de la confianza, y se diseñan escalas fiables y validas para medir la confianza y el compromiso de los clientes hacia la banca en internet.

\section{Análisis del compromiso y la confianza}

En esta sección, se realiza primero un análisis de la literatura referente al compromiso, y posteriormente se proponen las hipótesis referidas tanto a la composición de la confianza, como a la relación existente entre este concepto y el compromiso en el contexto de la banca en internet.

\subsection{Compromiso}

El compromiso ha sido considerado como uno de los conceptos centrales en el paradigma del marketing relacional (Dwyer et al., 1987; Morgan y Hunt, 1994; Wetzels et al., 2000).

Entre las definiciones más aceptadas de compromiso en la literatura académica de marketing, se encuentran la enunciada por Moorman et al. (1992, p.316), quienes lo definen como "un deseo duradero de mantener una relación que se valora", y la propuesta por Morgan y Hunt (1994, p.23), quienes siendo más específicos indican que el compromiso "es la creencia de un socio de intercambio que una relación con otro, es tan importante como para asegurar el máximo esfuerzo para mantenerla; esto es, la parte comprometida cree que vale la pena trabajar en la relación para asegurarse que dure indefinidamente".

Desde esta perspectiva, para que el compromiso este presente, es necesario que la relación sea lo suficientemente valiosa como para que las partes realicen todos los esfuerzos posibles para su mantenimiento (Morgan y Hunt, 1994, Wilson, 1995, Sharma y Patterson, 2000), llegando incluso a realizar sacrificios en el corto plazo para lograrlo (Anderson y Weitz, 1992; Siguaw et al., 1998). Según Dwyer et al. (1987), el compromiso, se basa fundamentalmente en el deseo permanente del consumidor por conservar una determinada relación y clasifican a este concepto como la etapa más alta en el vínculo relacional entre dos partes.

Los esfuerzos por intentar cuantificar el compromiso han sido numerosos y diversos, siendo considerado en muchas ocasiones como un constructo unidimensional (Morgan y Hunt, 1994; De Wulf y Odekerken-Schröder, 2003). No obstante lo anterior, y dependiendo del contexto y la ciencia que se involucra en su estudio, el compromiso ha tenido diversas propuestas como una estructura multi-dimensional, basada en un número no consensuado de sub-dimensiones que lo componen (Geyskens et al., 1996; Gundlach et al., 1995; Kumar et al.,1995).

La complejidad del concepto de compromiso ha generado divergencias en su concepción teórica, identificándose 
varias tipologías para definirlo y diversas conceptualizaciones de una o más dimensiones componentes (Sharma et al., 2006).

El compromiso ha sido comúnmente conceptualizado en marketing como una variable unidimensional (Garbarino y Johnson, 1999; Hennig-Thurau et al., 2002; Pritchard et al., 1999; Sharma y Patterson, 2000), contrastando con la perspectiva de otras áreas, como el comportamiento organizacional y la psicología social, que sugieren que es multidimensional (Adams y Jones, 1997; Meyer y Herscovitch, 2001; O'Reilly y Chatman, 1986). Sin embargo, en el último tiempo, son cada vez más los trabajos que ven al compromiso como multidimensional en esta área de estudio (Gilliland y Bello, 2002; Gundlach et al., 1995; Gruen et al., 2000).

En general, dentro de los estudios que consideran al compromiso como multidimensional, se ha utilizado una conceptualización de 3 sub-dimensiones que ha sido derivada de estudios en el campo de la psicología, éstas son, compromiso afectivo, calculado y normativo (Cater y Zabkar, 2008). De éstos, ha destacado en los estudios de marketing la utilización de los 2 primeros, afectivo y calculado (Geyskens et al., 1996), concentrando la mayor parte de los estudios del área (Gundlach et al., 1995; Kumar et al., 1995; Geyskens et al., 1996; Amine, 1998; Fullerton, 2005), salvo algunas excepciones (Kumar et al., 1994; de Ruyter y Semeijn, 2002; Bansal et al., 2004; Cater y Zabkar, 2008).

El compromiso afectivo está ligado al sentimiento emocional de cercanía entre las partes (Geyskens et al., 1996; Me- yer et al., 1993), asumiendo que el involucramiento e interés se da por la intención de tener una relación de largo plazo (Anderson y Weitz, 1992), luego, existe un deseo de continuar con la relación en el futuro y debido a que el individuo gusta de relacionarse con su contraparte, disfruta de la relación y crea un sentido de lealtad y pertenencia (Bansal et al., 2004). A su vez, el compromiso calculado tiene su origen en la consideración de los costos de cambio y los costos hundidos, o por la falta de alternativas válidas para establecer relaciones, así, el individuo genera compromiso porque mantener la relación actual representa menores costos que terminarla y comenzar una nueva (Casaló et al., 2007). Por su parte, el compromiso normativo se refiere a aquel que es obtenido al sentir la obligación de continuar con una relación, es decir, cuando hay un sentimiento de que la relación debe ser mantenida (Geyskens et al., 1996; Kumar et al., 1994).

Con base en lo anterior, podemos decir que los distintos tipos de compromiso tienen distintas motivaciones para mantener la relación, de esta forma, el compromiso afectivo indica que se "quiere" mantener la relación, el compromiso calculado indica que se "necesita" mantener la relación y el compromiso normativo indica que se "debe" mantener la relación (Bansal et al., 2004).

A pesar de las diversas estructuras multi-dimensionales propuestas para el compromiso en la literatura, gran cantidad de estudios lo han considerado como una variable unidimensional (Morgan y Hunt, 1994; de Wulf y OdekerkenSchröder, 2003). Lo anterior, se debe principalmente a que, de los componen- 
tes expuestos, sólo el compromiso afectivo tiene influencia en la intención del consumidor de querer mantenerse en una relación con la firma, por el contrario, el compromiso calculado es criticado por compartir el significado con intención conductual (Gundlach et al., 1995) y el compromiso normativo por contribuir a una relación de dependencia más que a una dedicación (Roberts et al., 2003). Además, se considera que el compromiso afectivo se desarrolla efectivamente a través del tiempo, debido a un conjunto de respuestas emocionales positivas, lo que lleva a estar cada vez más seguro de la relación (Stern, 1997). Todo lo anterior hace que compromiso afectivo sea considerado el componente más relevante y el único que tiene una influencia determinante y positiva en la generación de repetición voluntaria en el consumo, recomendación y por último, lealtad.

\subsection{Confianza}

La confianza es importante en la formación, desarrollo y mantención de las relaciones de marketing (Ganesan, 1994), y junto con el compromiso, son dos de los factores claves para mantener las relaciones de largo plazo (Morgan y Hunt, 1994).

Lo anterior, es un aspecto que ha suscitado gran interés por parte de los investigadores del área de marketing. Buena prueba de ello, es el gran número de trabajos en los que se ha abordado este concepto. De esta forma, podríamos ver como en algunos trabajos se ha estudiado el papel que la confianza juega en la conducta (Chaudhuri y Holbrook, 2001), en las comunicaciones entre los emplea- dos (Morgan y Hunt, 1994; Dahlstrom y Nygaard, 1995), en el desarrollo de las alianzas estratégicas (Morgan y Hunt, 1994; Smith y Barclay, 1997), en la relación comprador-proveedor (Morgan y Hunt, 1994; Doney y Cannon, 1997; Jap, 1999), en la relación empresa-cliente (Morgan y Hunt, 1994; Tax et al., 1998; Grayson y Ambler, 1999), en la relación vendedor-cliente (Dwyer et al., 1987; Czepiel, 1990; Beatty et al., 1996), en las relaciones de marketing industrial o en los canales de distribución (Kumar et al., 1995; Gruen y Shah, 2000), e incluso en la relación entre los usuarios y los investigadores de marketing (Moorman et al., 1992; Moorman et al., 1993).

A pesar del amplio número de estudios que se han realizado, en la literatura especializada de marketing, existe un cierto consenso en cuanto a que la confianza es la voluntad de contar con el intercambio de la otra parte, o socio, en quien se confía (Moorman et al., 1993), la que se genera a partir de una expectativa optimista acerca del resultado de dicho intercambio (Hosmer, 1995).

Si se observa esta perspectiva, la confianza no es un factor que se debería presentar con la misma intensidad en todos los sectores industriales. De hecho, esta se manifiesta con menor intensidad en aquellos sectores que por su naturaleza son más intangibles, como es por ejemplo, el sector de los servicios. Más aún, si la transacción se realiza por internet, que por naturaleza es un sistema que crea más desconfianza que los medios de transacción tradicionales (Gefen et al., 2003).

En esta línea, uno de los factores claves en el estudio de la confianza, es 
conocer las diferentes dimensiones que podrian forman parte de ella, dependiendo del sector en estudio. En la literatura se han identificado ampliamente tres dimensiones como parte de la confianza, éstas son: benevolencia, honestidad y competencia percibida. Dependiendo del sector, cada una de ellas ha contado con diferentes niveles de adhesión por partes de los investigadores. La benevolencia se refiere al reflejo de la motivación de una de las partes por anteponer el interés de la contraparte sobre el propio (Sirdeshmukh et al., 2002) imperando de esta manera el beneficio mutuo (Doney y Cannon, 1997). La honestidad en tanto, se refiere a la sinceridad y mantenimiento de las promesas de la contraparte (Doney y Cannon, 1997). Por último, la competencia percibida se refiere a las habilidades, capacidades y características que llevan a una parte a dominar una temática específica que es relevante para el éxito de la transacción (Mayer et al., 1995), o para satisfacer las necesidades de los clientes (Coulter y Coulter, 2002).

Como se puede apreciar en la literatura, la confianza es analizada mayoritariamente como una variable bidimensional, cuyos elementos constitutivos son la benevolencia y la honestidad (Geyskens et al., 1996; Doney y Cannon, 1997, Geyskens et al., 1998; Siguaw et al., 1998). Si consideramos que en el caso de la banca en internet, el medio de comunicación y transacción utilizado genera por naturaleza una percepción de riesgo elevada (Tan, 1999; Donthu y García, 1999), es razonable cuestionarse la real bidimensionalidad de la confianza. Bajo esta perspectiva, parece razonable agregar otra dimensión que haga referencia a las competencias percibida por los consumidores (Sirdesmukh et al., 2002; Suh y Han, 2003).

De hecho, la confianza hacia un banco en internet no solamente se fundamenta en la honestidad y la benevolencia, sino que además se basa en la percepción del consumidor, respecto a la capacidad técnica del banco para ofrecer sus servicios sin contratiempos, que pudieran generar desconfianza en el consumidor. Por tanto, en este contexto, no es garantía de confianza el que un banco realice promesas reflejando su honestidad o teniendo buenas intenciones para mostrarse benevolente, sino que es muy importante garantizar la seguridad, la privacidad y la efectividad de las transacciones que el cliente haga a través de internet.

Es así como, asumiendo los razonamientos anteriores, se puede suponer que en el sector bancario en internet, la confianza se compone de tres dimensiones representadas por la honestidad, benevolencia y competencia percibida. De acuerdo a lo anterior, se expone la siguiente hipótesis:

H1: El grado de confianza del usuario de la banca en internet es una variable multidimensional que se encuentra integrada por tres dimensiones básicas: honestidad, benevolencia y competencia percibida.

\subsection{Análisis de la relación entre la confianza y el compromiso}

Como se ha plateado en la literatura, una de las principales ventajas de la confianza es que permite entregar mayor 
valor a una relación, y a su vez, hacerla más estrecha (Chow y Holden, 1997; Macintosh et al, 1992; Doney y Cannon, 1997), hasta el punto que es capaz de generar algún grado de compromiso entre las partes (Moorman et al., 1992; Morgan y Hunt, 1994; Sharma y Patterson, 1999).

Ambos conceptos, confianza y compromiso han sido asociados en muchos estudios, principalmente en contextos que involucran la relación entre empresas (Anderson y Weitz, 1992; Ganesan, 1994). En la relación cliente-proveedor, el primero, al estar comprometido, está más dispuesto a dejar de lado beneficios inmediatos (de corto plazo) debido a la convicción más fuerte de un potencial beneficio futuro en la relación (de largo plazo). Luego, al estar más comprometido, existe un sentimiento de mayor vulnerabilidad, que implica que solo se comprometerá con aquellos vendedores en quienes confía (Walter y Ritter, 2003).

Para el estudio de la relación entre confianza-compromiso, ha sido habitual el uso de una conceptualización unidimensional del compromiso en la literatura de marketing, tomando como dimensión relevante el compromiso afectivo (Anderson y Weitz, 1992; Moorman et al., 1992; Morgan y Hunt, 1994; Geyskens et al., 1999; Garbarino y Johnson, 1999; Grayson y Ambler, 1999; Goodman y Dion, 2001; Perry et al., 2004; Tellefsen y Thomas, 2005; Eastlick et al., 2006; Casaló et al., 2007). En los casos en que se presenta una estructura bidimensional del compromiso (incluyendo compromiso afectivo y calculado), la propuesta teórica presenta un efecto de la confianza sólo como determinante del compromiso afectivo (Li et al., 2006; N'Goala, 2007). Por su parte, quienes han propuesto la relación hacia ambos tipos de compromiso, han encontrado una relación positiva entre confianza y compromiso afectivo y una relación negativa entre confianza y compromiso calculado (Geyskens et al., 1996; de Ruyter y Wetzels, 1999; de Ruyter et al., 2001; Gounaris, 2005). Es más, estudios recientes han probado que proponiendo teóricamente un efecto de la confianza sobre los 3 tipos de compromiso (afectivo, calculado y normativo), es sólo el afectivo el que presenta un efecto significativo, desechando las otras dos relaciones (Cater y Zabkar, 2008).

En el caso específico de la relación entre personas y proveedores de servicios a través de internet, cada vez se agrega mayor evidencia que apunta a comprobar una relación directa entre confianza y compromiso, sobre la base de una propuesta de compromiso unidimensional (Wagner y Rydstrom, 2001; Bauer et al., 2002; Mukherjee y Nath, 2003; Casaló et al., 2007).

Es bajo este esquema, que en la banca a través de internet se espera que la confianza cumpla un papel importante en la disminución del riesgo y en la posterior generación del compromiso del consumidor hacia la relación con su proveedor de servicios (Suganthi et al., 2001). De esta forma, de acuerdo a los argumentos precedentes, se propone la siguiente hipótesis:

$\mathrm{H}_{2}$ : En el contexto de la banca en internet, mientras mayor sea la confianza del cliente, mayor será el nivel de compromiso hacía el proveedor de servicios. 


\section{Construcción de las escalas de medida y obtención de los Datos}

Para desarrollar las escalas con suficiente grado de validez de contenido, se consideró como base en el caso de la confianza, las escalas desarrolladas por Doney y Canon (1997). En el caso del compromiso se consideró las escalas de Moorman et al. (1992) y Anderson y Weitz (1992). El punto de partida fue una escala multidimensional en el caso de la confianza, compuesta por las sub-dimensiones honestidad, benevolencia y competencia. Por su parte, siguiendo el planteamiento de Roberts et al., (2003), sólo se consideró el componente afectivo en el estudio del compromiso, alternativa que ha sido seguida por diversos estudios recientes en el área de marketing (Perry et al., 2004; Eastlick et al., 2006; Casaló et al., 2007).

En un paso posterior, se depuraron las escalas previas en base a sucesivos focus groups con clientes que han realizado operaciones financieras con un banco a través de internet y diversas entrevistas en profundidad con ejecutivos de bancos que operan por este canal. Estos análisis permitieron por un lado, agregar aquellos indicadores que reflejen más adecuadamente la confianza y el compromiso en la banca en internet, y por otro lado readecuar y/o eliminar aquellos indicadores que son conflictivos o redundantes para los conceptos analizados. Con este proceso se obtuvieron las primeras escalas con las que se construyó la primera versión del cuestionario a ser aplicado.

Finalmente, con el cuestionario inicial se realizó un pre-test cuantitativo a una muestra de 40 personas que ha reali- zado operaciones con un banco a través de internet. Posteriormente con esos datos se realizó un análisis factorial exploratorio y se calculó el alpha de Cronbach a cada una de las dimensiones resultantes. Con este análisis previo se pudo confirmar la existencia de cada una de las dimensiones propuestas y sus respectivos ítems.

Todas las preguntas fueron redactadas como afirmaciones, donde los encuestados debían responder a través de una escala Likert de 1 (totalmente en desacuerdo) a 7 (totalmente de acuerdo) (ver Cuadro 1).

El estudio de campo fue realizado en Santiago de Chile entre los meses de agosto y septiembre de 2007. La entrevista personal fue realizada por teléfono a un total de 274 personas, quienes, como requisito, debían haber realizado a lo menos tres operaciones financieras mensuales a través de la página web de su banco, durante los últimos tres meses. Para esto, se utilizó un software especializado que permite seleccionar de manera aleatoria tanto el teléfono a contactar, como el orden de las preguntas Likert a ser planteadas a los entrevistados. Utilizar el formato telefónico, permitió contar con un marco muestral representativo del universo de estudio.

El $46 \%$ de los entrevistados fueron mujeres y el $54 \%$ hombres. El $18 \%$ tenía una edad entre 18 y 22 años, el $34 \%$ entre 23 y 32 años, el $20 \%$ entre 33 y 42 años, el $15 \%$ entre 43 y 52 años, el $8 \%$ entre los 53 y 64 años, y el $3 \%$ con una edad mayor a los 64 años (un $2 \%$ no respondió a esta pregunta). Por otra parte, el $10 \%$ percibía un ingreso familiar de hasta 13.000.- dólares al año, el 20\% percibía entre 


\section{Cuadro 1 \\ Escalas de confianza y compromiso}

Honestidad

Mi banco suele cumplir los compromisos que asume a través de internet.

La atención de mi banco a través de internet se caracteriza por ser sincera y honesta.

Puedo fiarme de las promesas de mi banco a través de internet.

Benevolencia

Las recomendaciones y advertencias dadas por mi banco a través de internet son realizadas en busca del beneficio mutuo.

Mi banco a través de internet se preocupa de los intereses presentes y futuros de sus clientes.

Mi banco tiene en cuenta las consecuencias que sus acciones podrían tener en sus clientes de internet.

El diseño y variedad de productos que mi banco ofrece a través de internet tiene en cuenta los deseos y necesidades de sus clientes.

Mi banco a través de internet es receptivo a las necesidades de sus usuarios.

\section{Competencia}

Mi banco tiene habilidades para llevar a cabo las operaciones a través de internet.

Mi Banco tiene los recursos necesarios para realizar con éxito sus actividades en internet.

Mi banco es competente y efectivo en realizar operaciones a través de internet.

\section{Compromiso}

La relación con mi banco a través de internet, es algo que pretendo mantener en el futuro

Estoy muy comprometido con mi banco.

La relación que tengo con mi banco a través de internet merece el mayor esfuerzo para mantenerla.

Fuente: elaboración propia.

13.001.- y 20.000.- dólares, el $36 \%$ entre 20.001.- y 41.000 dólares y el $30 \%$ más de 41.000.- dólares al año (el $4 \%$ no respondió a esta pregunta). Finalmente, el $4 \%$ tenía estudios de postgrado, el $65 \%$ tenía carrera universitaria, el $22 \%$ tenía estudios de educación superior técnica y el $9 \%$ tenía sólo estudios secundarios.

\section{Depuración de las escalas y contraste de las hipótesis}

Una vez construidas las escalas de confianza y compromiso, con buen grado de validez de contenido, y obtenidos los datos a través de entrevistas personales, el siguiente paso consistió en depurar las escalas de medidas propuestas. Para esto, en primer lugar se realizó un análisis exploratorio de fiabilidad y dimensionalidad de cada una de las sub-escalas que forman parte de la confianza y el compromiso hacia los bancos en internet. Posteriormente, se realizó un análisis confirmatorio de fiabilidad para ratificar que cada uno de los ítems formaba parte de cada una de las sub-escalas y así comprobar su dimensionalidad. Este último análisis, permitió contrastar la hipótesis 1. Luego, se midió el grado de validez de las esca- 
las de medida resultantes que permiten medir la confianza y el compromiso en la banca en internet. Finalmente, se desarrollo el modelo causal con el cual se contrastó la segunda hipótesis por medio de ecuaciones estructurales.

\subsection{Análisis exploratorio}

En esta sección se presenta un análisis exploratorio de fiabilidad y dimensionalidad de los constructos confianza y compromiso.

\section{a) Análisis de fiabilidad}

Con este análisis exploratorio se pretende eliminar todos aquellos ítems que impiden a las sub-escalas estudiadas alcanzar un nivel adecuado de fiabilidad. Para ello, se utilizó el estadístico alpha de Cronbach (Cronbach, 1951). En la literatura de análisis multivariado de datos, se plantea que para un análisis confirmatorio, es deseable un valor de alpha de Cronbach mayor a 0,7 (Nunally, 1978; Hair et al., 1998).

En cualquier caso, la fiabilidad alpha de una escala puede ser mejorada eliminando aquellos ítems cuya correla- ción con el resto de indicadores de la escala sea menor a un cierto umbral establecido. En este caso se consideró el planteamiento de Norusis (1993) que establece en 0,3 el nivel mínimo aceptado para este tipo de análisis.

Una vez aplicado el análisis de alpha de Cronbach a las tres sub-escalas de confianza y a la escala de compromiso, podemos apreciar (ver Tabla 1) que todas ellas superan el umbral de 0,7. Además se puede apreciar, que en todos los casos, las correlaciones ítems-total sub-escala superan considerablemente el límite establecido de 0,3. Por lo tanto, en esta etapa no fue necesario eliminar indicadores de las escalas propuestas.

\section{b) Análisis de dimensionalidad}

En esta parte del análisis se efectuó un estudio de dimensionalidad, que consiste en probar si cada sub-escala representa un único factor, es decir, si cada una de ellas tiene suficiente identidad como para representar una única variable latente (Flavián y Lozano, 2003). Para esto se utilizó el análisis factorial de componentes principales con rotación varimax (Hair et al., 1998).

\section{Tabla 1}

Alpha de Cronbach

\begin{tabular}{|c|c|c|c|c|c|c|c|}
\hline \multicolumn{2}{|c|}{ Honestidad } & \multicolumn{2}{|c|}{ Benevolencia } & \multicolumn{2}{|c|}{ Competencia } & \multicolumn{2}{|c|}{ Compromiso } \\
\hline \multicolumn{8}{|c|}{ Correlaciones ítem-total sub-escala } \\
\hline & & Benevo1 & 0.68 & & & & \\
\hline Honest1 & 0.84 & Benevo2 & 0.79 & Compet1 & 0.61 & Compr1 & 0,63 \\
\hline Honest2 & 0.89 & Benevo3 & 0.78 & Compet2 & 0.55 & Compr2 & 0,55 \\
\hline \multirow[t]{2}{*}{ Honest3 } & 0.80 & Benevo4 & 0.75 & Compet3 & 0.63 & Compr3 & 0,73 \\
\hline & & Benevo5 & 0.82 & & & & \\
\hline \multicolumn{8}{|c|}{ Alpha de Cronbach } \\
\hline \multicolumn{2}{|c|}{0,92} & \multicolumn{2}{|c|}{0,91} & \multicolumn{2}{|c|}{0,76} & \multicolumn{2}{|c|}{0,77} \\
\hline
\end{tabular}

Fuente: elaboración propia. 
Como paso previo al análisis de la dimensionalidad de las sub-escalas y como requisito previo para aprobar la utilización en el estudio del análisis factorial de componentes principales, se realizaron los test de Kaiser, Meyer y Olkin (KMO) y la prueba de Esfericidad de Bartlett (Hair et al., 1998). En todos los casos en que se utilizó el análisis factorial exploratorio, ambos test se encontraban dentro de los parámetros óptimos, lo que mostró la conveniencia de utilizar tal metodología para los fines de este análisis.

Los resultados muestran que en todos los casos las sub-escalas presentan un buen grado de dimensionalidad. Los indicadores correspondientes en cada caso resultaron reunidos en un único factor con una varianza explicada relativamente alta (ver Tabla 2).

\subsection{Análisis confirmatorio de fiabilidad y dimensionalidad}

A continuación se expone el análisis confirmatorio de fiabilidad y de dimensionalidad realizado a los constructos confianza y compromiso.

\section{a) Análisis confirmatorio de fia- bilidad}

En esta etapa se efectuó un análisis más exhaustivo de las escalas de confianza y compromiso, a través de un proceso de validación que permite representar un modelo teórico constituido por diferentes variables latentes y por un conjunto de ítems o indicadores que permitan medirlos. La herramienta más adecuada para representar este tipo de modelos, es el análisis factorial confirmatorio que es realizado a través del método de ecuaciones estructurales (Luque, 2000).

Para esto se utilizó el paquete estadístico EQS en su versión 5.7b. Por medio de este programa, se pudo estimar los parámetros del modelo mediante el método de máxima verosimilitud robusto $\mathrm{Hu}$ et al., 1992) que permite prevenir los posibles problemas derivados de la falta de normalidad univariada de los datos incluidos en el estudio.

A partir del desarrollo de un modelo compuesto por diferentes variables latentes que representan el constructo confianza, se llevó a cabo un proceso de mejora mediante una estrategia de desarrollo de modelo (Hair et al., 1998), que con-

Tabla 2

Análisis Factorial Exploratorio

\begin{tabular}{|c|c|c|c|c|c|c|c|}
\hline \multicolumn{2}{|c|}{ Honestidad } & \multicolumn{2}{|c|}{ Benevolencia } & \multicolumn{2}{|c|}{ Competencia } & \multicolumn{2}{|c|}{ Compromiso } \\
\hline Indicador & Carga & Indicador & Carga & Indicador & Carga & Indicador & Carga \\
\hline & & Benevo1 & 0.785 & & & & \\
\hline Honest1 & 0.931 & Benevo2 & 0.872 & Compet1 & 0.836 & Compr1 & 0.859 \\
\hline Honest2 & 0.955 & Benevo3 & 0.867 & Compet2 & 0.790 & Compr2 & 0.772 \\
\hline \multirow[t]{2}{*}{ Honest3 } & 0.909 & Benevo4 & 0.845 & Compet3 & 0.851 & Compr3 & 0.910 \\
\hline & & Benevo5 & 0.894 & & & & \\
\hline \multicolumn{2}{|c|}{ Varianza explicada } & \multicolumn{2}{|c|}{ Varianza explicada } & \multicolumn{2}{|c|}{$\begin{array}{c}\text { Varianza explicada } \\
68.25 \%\end{array}$} & \multicolumn{2}{|c|}{$\begin{array}{c}\text { Varianza explicada } \\
72.04 \%\end{array}$} \\
\hline
\end{tabular}

Fuente: Elaboración propia. 
siste en eliminar aquellos indicadores o variables menos adecuadas para alcanzar un buen ajuste de éstos.

Esta eliminación de indicadores que va generado diferentes modelos, es decir, diferentes alternativas con mejor ajuste del modelo teórico, se realiza considerando los criterios propuestos por Jöreskog y Sörbom (1993).

Los resultados del análisis factorial confirmatorio mostraron que en el caso del constructo confianza no fue necesario eliminar indicadores. De hecho, en todos los casos los indicadores mostraron una fuerte convergencia con su correspondiente variable latente, coeficientes estandarizados mayores a 0,5 y $R^{2}$ mayores a 0,3 (Diagrama 1). Además, el modelo mostró indicadores de ajuste dentro de los niveles aceptables (ver Tabla 3).

\section{Tabla 3}

Ajustes del Modelo Confirmatorio de Confianza

\begin{tabular}{cc}
\hline IFI & 0,911 \\
CFI & 0,910 \\
RMSR & 0,153 \\
AIC & 58,88 \\
\hline
\end{tabular}

Fuente: elaboración propia.

b) Dimensionalidad del constructo confianza

Con el objeto de determinar si el constructo confianza está compuesto por diferentes dimensiones de acuerdo a como los platea la hipótesis 1 , se desarrollo una Estrategia de Modelos Rivales (Hair et al., 1998). De esta manera se contrastó si la confianza en el contexto de la banca en internet está compuesta por

\section{Análisis Confirmatorio del Constructo Confianza}

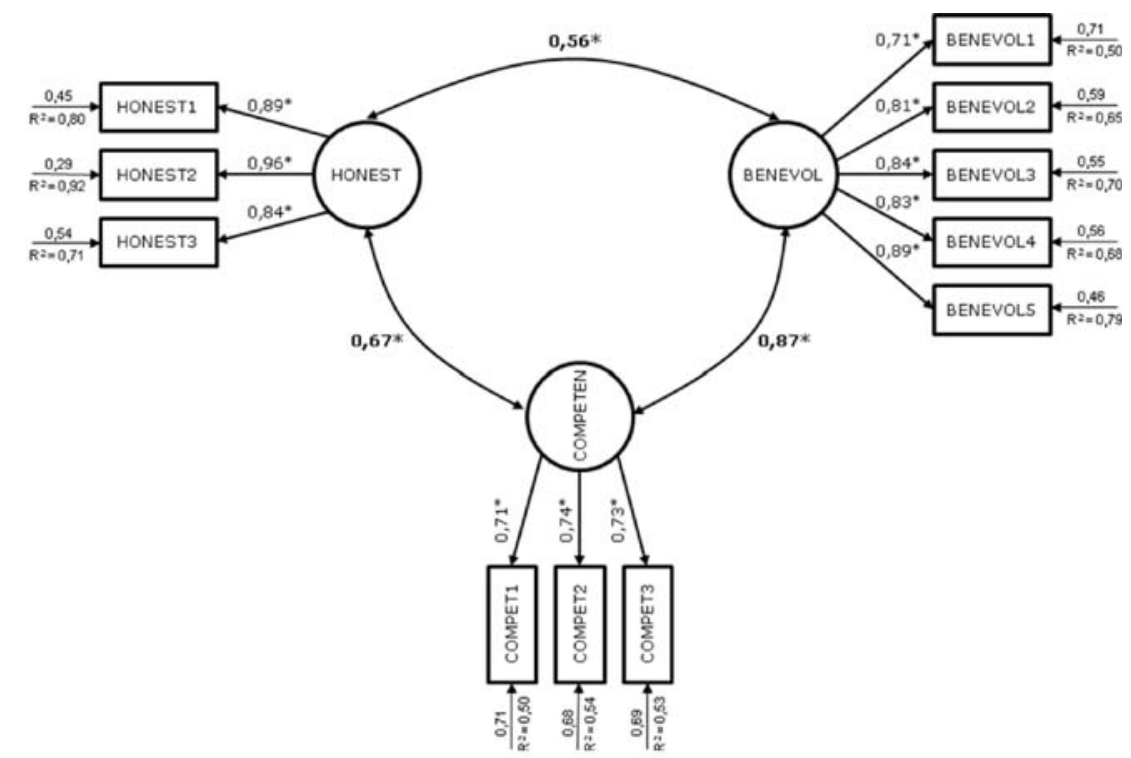

Fuente: elaboración propia. 
las dimensiones honestidad, benevolencia y competencia. Este contraste de modelos rivales se llevó a cabo comparando un modelo de primer orden, es decir uno en el que todos los indicadores o ítems del constructo cargan sobre un solo factor, con otro de segundo orden donde los constructos tanto de la confianza como el compromiso están representados por diferentes dimensiones.

En la Tabla 4 se pueden apreciar los resultados que indican que en el caso de la confianza el modelo de segundo orden presentó mejores indicadores de ajustes que el de primer orden. De esta manera la confianza en el contexto de la banca en internet muestra ser una variable multidimensional, lo que respalda la hipótesis 1.

\subsection{Análisis finales de fiabilidad}

Considerando la confianza en la banca en internet como una variable multidimensional y el compromiso como unidimensional, es necesario finalmente confirmar la fiabilidad de las sub-escalas propuestas utilizando además del alpha de Cronbach otros dos criterios como son el análisis de fiabilidad compuesta del constructo y el análisis de varianza extraída.

Al no haber eliminación de elementos entre los ítems de cada uno de los constructos estudiados, los valores del indicador Alpha de Cronbach se mantuvieron respecto de lo señalado inicialmente (ver Tabla 1).

Al aplicar la fiabilidad compuesta del constructo, todas dimensiones de la confianza y el constructo compromiso resulta-

\begin{tabular}{ccc}
\multicolumn{2}{c}{ Análisis $\begin{array}{c}\text { Tabla } 4 \\
\text { Multidimensional }\end{array}$} \\
\cline { 2 - 3 } & \multicolumn{2}{c}{ Confianza } \\
\cline { 2 - 3 } Primer orden & Segundo orden \\
\hline IFI & 0,702 & 0,910 \\
CFI & 0,699 & 0,909 \\
RMSR & 0,221 & 0,152 \\
AIC & 288,83 & 61,09 \\
\hline
\end{tabular}

Fuente: elaboración propia.

ron con valores igual o sobre el mínimo establecido para este indicador que es de 0,70 (honestidad 0,85 ; benevolencia 0,85 , competencia 0,70 y compromiso 0,75 ).

Finalmente, en el caso del análisis de la varianza extraída, los valores arrojados para cada dimensión estudiada fueron: 0,65 para honestidad, 0,54 para benevolencia, 0,43 para competencia y 0,51 para compromiso.

Con lo anterior, es posible pensar que cada uno de los constructos propuestos tiene un grado suficiente de fiabilidad, la cual está garantizada por el análisis conjunto de los índices Alpha de Cronbach, Fiabilidad Compuesta del Constructo y Varianza Extraída.

\subsection{Análisis de validez}

Para medir la validez, se siguió el esquema planteado por Nunnally (1978). Según este autor para que exista validez debe comprobarse que la escala empleada tenga validez de contenido, validez de constructo y validez con relación a un criterio.

Con respecto a la validez de contenido, está en un buen grado garantizada, debido a que las escalas utilizadas para 
medir tanto la confianza como el compromiso en el contexto de la banca en inter$n e t$, se han diseñado a partir de un análisis detallado de la literatura, posteriormente, han sido sometidas al juicio y a la discusión de diferentes expertos y han sido objeto de un proceso de depuración posterior.

Para llevar a cabo el análisis de validez de constructo, fue preciso comprobar si las sub-escalas de confianza resultantes del análisis factorial confirmatorio superaban las pruebas de validez convergente y validez discriminante (estas pruebas no serán aplicadas al factor compromiso, debido a que éste es unidimensional y por lo tanto, la validez discriminante no aplica y la condición la validez convergente está garantizada). El modelo de confianza, cumplirá la condición de validez convergente si las variables latentes que la componen están fuerte y significativamente correlacionadas entre ellas. Al observar el diagrama 1 que representa el modelo resultante del proceso de depuración realizado mediante el análisis factorial confirmatorio, podemos constatar que las correlaciones entre las variables latentes que componen la escala de confianza, son en todos los casos relativamente elevadas y significativas al $0,01$ ( $t>2,58)$, por lo que podemos demostrar que existe validez convergente.
Por su parte las escalas propuestas tendrán validez discriminante, si los factores que se han utilizado para medirlas no sirven para medir otros constructos, o dicho de otro modo, se correlacionan más entre sí que con otros factores 0 variables latentes que están menos relacionados con el concepto estudiado. Para comprobar la existencia de validez discriminante se utilizó el test del intervalo de confianza (Anderson y Gerbing, 1988).

El test de intervalos de confianza indica que existe validez discriminante entre dos variables latentes, cuando en los intervalos de confianza resultantes de estimar la correlación entre ambas variables latentes, no se encuentra incluido el valor "1". En la Tabla 5 se puede apreciar, que en ningún caso se encuentra contenido el valor "1". Es más, en todos los casos, las correlaciones se alejan considerablemente de este valor.

Por lo tanto, con todos estos antecedentes estamos en condiciones de afirmar que en este modelo de confianza propuesto en el contexto de la banca en internet, existe validez discriminante entre las variables latentes que la integran.

Una vez realizados los análisis para determinar la validez interna a través de la validez de contenido y de constructo, es preciso efectuar un análisis ex-

Tabla 5

Test de Validez Discriminante del Constructo Confianza

Pares de Constructos

Honestidad - Benevolencia

Honestidad - Competencia

Benevolencia - Competencia
Confidence Intervals

0,$425 ; 0,689$

0,$545 ; 0,737$

0,$786 ; 0,954$

Fuente: elaboración propia. 
terno del modelo, por medio del análisis de validez con relación a un criterio.

Este análisis tiene como principal propósito, conocer con qué eficacia el concepto analizado se relaciona con aquellas variables criterio con los que la teoría indica que deben estar relacionadas. Desde el punto de vista del momento en el tiempo en que se obtienen las variables analizadas, se pueden realizar dos tipos de análisis de validez con relación a un criterio: validez concurrente y validez predictiva. Como los datos han sido obtenidos en un mismo momento del tiempo no podemos hacer validez predictiva y por to tanto realizamos validez concurrente. Según la literatura especializada de marketing se espera que la confianza tenga una fuerte relación con el compromiso, análisis que se llevará cabo en el próximo epígrafe donde se contrastará empíricamente la hipótesis 2 y se podrá conocer la validez concurrente del constructo confianza que ha sido propuesto en este modelo.

\subsection{Análisis de la relación confianza-compromiso}

Ahora que contamos con escalas de confianza y compromiso con un buen grado de fiabilidad, validez y dimensionalidad, estamos en condiciones de analizar si la confianza es un factor que influye sobre el compromiso en el contexto de la banca en internet, de acuerdo a como se plantea en la hipótesis 2.

En el Diagrama 2 se puede observar que el parámetro estandarizado de la relación de la confianza sobre el compromiso resulta positivo y significativo al 0,01 . Además el modelo tiene una alta capacidad explicativa $\left(R^{2}\right.$ de 0,85$)$. Por lo tanto, con todos estos antecedentes se puede respaldar la hipótesis 2 .

Por otro lado, es necesario destacar que todos los indicadores superan los parámetros establecidos por Jöreskog y Sörbom (1993). Es decir, en todos los casos se presenta una alta significancia de los coeficientes de regresión factorial entre los ítems y su correspondiente variable latente. Además, en todos ellos las cargas de las variables traducidas en coeficientes estandarizados fueron mayores a 0,5 (valor mínimo $=0,65$ ) y todas las variables presentaron un $\mathrm{R}^{2}$ mayor a 0,3 (valor mínimo $=0,42$ ).

Finalmente, como se puede observar en la Tabla 6 , los niveles de ajuste del modelo están dentro de los valores óptimos.

\section{Diagrama 2}

Relación Causal Confianza-Compromiso

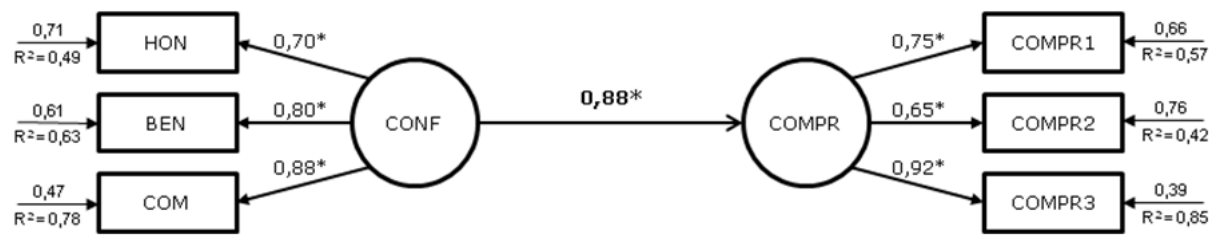

Nota: * significativo al 0.01 .

Fuente: elaboración propia. 


\section{Tabla 6 \\ Ajustes del Modelo \\ Confianza-Compromiso}

\begin{tabular}{cc}
\hline IFI & 0,939 \\
CFI & 0,938 \\
RMSR & 0,109 \\
AIC & 62,454 \\
\hline
\end{tabular}

\section{Conclusiones e implicaciones para la gestión}

Este estudio considera como punto de partida, que lograr el compromiso de los clientes hacia un banco en internet es un importante desafío para estas instituciones financieras, y que al igual que en otros contextos, la confianza podría cumplir un importante papel en la consecución de este objetivo.

Sin embargo, la confianza podría estar compuesta por diferentes factores, lo que podría traer importantes implicaciones para la gestión de esta variable, y para alcanzar en consecuencia el compromiso de los clientes con su banco en internet.

Los resultados de este estudio permitieron determinar, que en efecto, la confianza es un importante generador del compromiso de los clientes hacia su banco en internet. Los resultados también mostraron, que en este contexto bancario, la confianza se comporta como un constructo multidimensional compuesto por tres dimensiones, como son la honestidad, la benevolencia y la competencia.

Estos hallazgos traen importantes implicaciones para la gestión de este tipo de bancos. De hecho, para que los bancos en internet logren el compromiso de sus clientes, deberían centrar la gestión de la confianza del cliente, en cada una de las dimensiones que la componen. En concreto, los bancos deben siempre cumplir con las promesas que realizan a sus clientes a través de sus páginas web. Además, deben buscar el beneficio mutuo en la transacción que realizan, sin mostrar un comportamiento oportunista hacia sus clientes. Finalmente, deberían ser competentes y eficientes en la gestión de las relaciones con los clientes a través de la web. Es decir, centrar sus esfuerzos, en mantener una página web con buenos niveles de usabilidad, donde los clientes puedan navegar de manera fácil y realizar sus transacciones rápidamente y sin problemas. Además, los bancos deberían mantener los datos financieros y personales de sus clientes con total exactitud. De la misma manera, deberian tener un sistema de seguridad en la web que garantice la privacidad de los datos y de las transacciones realizadas. Por último, deberían ofrecer productos y servicios atractivos a sus clientes, con costos financieros acordes a la industria.

A pesar de que el banco debe centrar su gestión en estas tareas, esto no es suficiente para lograr la confianza y el posterior compromiso de sus clientes. Es imprescindible que el banco sea percibido por sus clientes como una institución financiera honesta, benevolente y competente. Es decir, no es suficiente con el banco se comporte consecuente con estos tres pilares de la confianza, sino que es muy importante que los clientes asocien al banco con estas buenas prácticas.

Desde esta perspectiva, los directivos del banco deben centrar sus esfuerzos no sólo en las prácticas de gestión del banco, sino que también en las herra- 
mientas promocionales de marketing que permiten que estos factores generadores de confianza sean percibidos como parte constitutiva de la filosofía del banco.

\section{Referencias bibliográficas}

Adams, Jeffrey M. y Jones, Warren H. (1997). The Conceptualization of Marital Commitment: An Integrative Analysis, Journal of Personality and Social Psychology, 72 (5), USA, American Psychological Association, pp. 11771196.

Amine, Abdelmajid (1998), Consumers' True Brand Loyalty: The Central Role of Commitment, Journal of Strategic Marketing, 6 (4), UK, Routledge, pp. 305-319.

Anderson, James C. y Gerbing, David W. (1988), Structural Equation Modelling in Practice: A Review and Recommended Two-Step Approach, Psychological Bulletin, 103 (3), USA, American Psychological Association pp. 411-423.

Anderson, Erin y Weitz, Barton (1992), The Use of Pledges to Build and Sustain Commitment in Distribution Channels, Journal of Marketing Research, 29 (February), USA, American Marketing Association, pp. 18-34.

Bansal, Harvir S.; Irving, P. Gregory y Taylor, Shirley F. (2004), A Three-Component Model of Customer Commitment to Service Providers, Journal of the Academy of Marketing Science, 32 (3), USA, Academy of Marketing Science, pp. 234-250.

Bauer, Hans H.; Grether, Mark y Leach, Mark (2002), Building Customer Relations over the Internet, Industrial Marketing Management, 31 (2), UK, Elsevier, pp. 155-163.
Beatty, Sharon E.; Mayer, Morris; Coleman, James E.; Reynolds, Kristy E. y Lee, Jungki (1996), Customer-Sales Associate Retail Relationships, Journal of Retailing, 72 (3), USA, Elsevier, pp. 223-247.

Casaló, Luis V.; Flavián, Carlos y Guinalíu, Miguel (2007), The Influence of Satisfaction, Perceived Reputation and Trust on a Consumer's Commitment to a Website, Journal of Marketing Communications, 13 (1), UK, Routledge, pp. 1-17.

Cater, Barbara y Zabkar, Vesna (2008), Antecedents and Consequences of Commitment in Marketing Research Services, Industrial Marketing Management, doi: 10.1016/j.indmarman. 2007.10.004, USA, Elsevier.

Chaudhuri, Arjun y Holbrook, Morris B. (2001), The Chain of Effects from Brand Trust and Brand Affect to Brand Performance: The Role of Brand Loyalty, Journal of Marketing, 65 (2), USA, American Marketing Association, pp. 81-93.

Chow, Simeon y Holden, Reed (1997), Toward an Understanding of Loyalty: The Moderating Role of Trust, Journal of Managerial Issues, Vol. 9 (3), USA, Pittsburg State University pp. 275299.

Coulter, Kevin S. y Coulter, Robin A. (2002), Determinants of Trust in a Service Provider: The Moderating Role of Length of Relationship, Journal of Services Marketing, 16 (1), UK, Emerald Publishing, pp. 35-50.

Cronbach, Lee J. (1951), Coefficient Alpha and the Internal Structure of Test, Psichometrica, 16 (October), USA, Springer, pp. 297-334.

Czepiel, John A. (1990), Service Encounters and Service Relationships: Implications for Research, Journal of Busi- 
ness Research, 20, USA, Elsevier, pp. 13-21.

Dahlstrom, Robert y Nygaard, Arne (1995): An Exploratory Investigation of Interpersonal Trust in New and Mature Market Economies, Journal of Retailing, 71 (Winter), USA, Elsevier, pp. 339-361.

de Ruyter, Ko; Moorman, Luci y Lemmink, Jos (2001), Antecedents of Commitment and Trust in Customer-Supplier Relationships in High Technology Markets, Industrial Marketing Management, Vol. 30, No. 3, USA, Elsevier, pp. 271-286.

de Ruyter, Ko y Semeijn, Janjaap (2002), Forging Buyer-Seller Relationships for Total Quality Management in International Business: The Case of European Cement Industry, Total Quality Management, 13(3), UK, Routledge, pp. 403-417.

de Ruyter, Ko y Wetzels, Martin (1999), Commitment in Auditor-Client Relationships: Antecedents and Consequences, Accounting, Organizations and Society, 24 (1), USA, Elasevier, pp. 57-75.

de Wulf, Kristof y Odekerken-Schröder, Gaby (2003), Assessing the Impact of a Retailer's Relationship Efforts on Consumer' Attitudes and Behaviour, Journal of Retailing and Consumer Services, 10, UK, Elsevier, pp. 95108.

Doney, Patricia M. y Cannon, Joseph P. (1997), An Examination of the Nature of Trust in Buyer-Seller Relationships, Journal of Marketing, 61 (2), USA, American Marketing Association, pp. 35-51.

Donthu, Naveen y Garcia, Adriana (1999), The Internet Shopper, Journal of Advertising Research, 39, USA, Advertising Research Foundation, pp. 52-58.

Dwyer, F. Robert; Schurr, Paul H. y Oh, Sejo (1987), Developing Buyer-Seller Re- lationships, Journal of Marketing, 51 (2), USA, American Marketing Association, pp. 11-25.

Eastlick, Mary A.; Lotz, Sherry L. y Warrington, Patricia (2006), Understanding Online B-to-C Relationships: An Integrated Model of Privacy Concerns, Trust, and Commitment, Journal of Business Research, 59, USA, Elsevier, pp. 877-886.

Flavián, Carlos y Lozano, Francisco J. (2003): Diseño de una Escala para Medir la Orientación al Entorno de la Nueva Formación Profesional, Cuadernos de Economía y Dirección de Empresas, España, ACEDE, 15, pp. 333-363.

Fullerton, G. (2005), The Impact of Brand Commitment on Loyalty to Retail Service Brands, Canadian Journal of Administrative Sciences, 22(2), Canadá, Revue Canadienne des Sciences de l'Administration, pp. 97-110.

Ganesan, Shankar (1994), Determinants of Long-Term Orientation in Buyer-Seller Relationship, Journal of Marketing, 58(2), USA, American Marketing Association, pp. 1-19.

Garbarino, Ellen y Johnson, Mark S. (1999). The Different Roles of Satisfaction, Trust, and Commitment in Customer Relationships, Journal of Marketing, 63, USA, American Marketing Association, pp. 70-87.

Gefen, David; Karahanna, Elena y Straub, DetmarW. (2003), Trust and TAM in Online Shopping: An Integrated Model, MIS Quarterly, 27(1), MIS Research Center, pp. 51-90.

Geyskens, Inge; Steenkamp, Jan B. y Kumar, Nirmalya (1998), Generalizations About Trust in Marketing Channel Relationships Using Meta-Analysis, International Journal of Research in Marketing, 15, Holanda, Elsevier, pp. 223-248. 
Geyskens, Inge; Steenkamp, Jan B. y Kumar, Nirmalya (1999), A Meta-Analysis of Satisfaction in Marketing Channel Relationships, Journal of Marketing Research, 36(2), USA, American Marketing Association, pp.223-238.

Geyskens, Inge; Steenkamp, Jan B.; Scheer, Lisa K. y Kumar, Nirmalya (1996), The Effects of Trust and Interdependence on Relationship Commitment: a Trans-Atlantic study, International Journal of Research in Marketing, 13, Holanda, Elsevier, pp. 303-317.

Gilliland, David I. y Bello, DanielC. (2002), Two Sides to Attitudinal Commitment: The Effects of Calculative and Loyalty Commitment on Enforcement Mechanism in Distribution Channels, Journal of the Academy of Marketing Science, 30(1), USA, Academy of Marketing Science, pp. 24-43.

Goodman, Lester E. y Dion, Paul A. (2001), The Determinants of Commitment in the Distributor-Manufacturer Relationship, Industrial Marketing Management, 30(3), USA, Elsevier, pp. 287-300.

Gounaris, Spiros P. (2005), Trust and Commitment Influences on Customer Retention: Insights from Business-to-Business Services, Journal of Business Research, 58(2), USA, Elsevier, pp. 126-140.

Grayson, Kent y Ambler, Tim (1999), The Dark Side of Long-Term Relationships in Marketing Services, Journal of Marketing Research, 36, USA, American Marketing Association, pp. 132-141.

Gruen, Thomas W. y Shah, Reshma H. (2000): Determinants and Outcomes of Plan Objectivity and Implementation in Category Management Relationships, Journal of Retailing, 76(4), USA, EIsevier, pp. 483-510.

Gruen, T.W.; Summers, John O. y Acito, Frank (2000), Relationship Marketing Activi- ties, Commitment, and Membership Behaviors in Professional Associations, Journal of Marketing, 64, USA, American Marketing Association, pp. 34-49.

Gundlach, Gregory T.; Achrol, Ravi S. y Mentzer, John M. (1995), The Structure of Commitment in Exchange, Journal of Marketing, 59, USA, American Marketing Association, pp. 78-92.

Hair, Joseph F. Jr., Anderson, Rolph E., Tatham, Ronald L. and Black, William C. (1998), Multivariate data analysis, Prentice Hall, 5th edition.

Hennig-Thurau, Thorsten; Gwinner, Kevin P. y Gremler, Dwayne D. (2002), Understanding Relationship Marketing Outcomes, Journal of Service Research, 4(3), USA, Sage Publications, pp. 230-247.

Hosmer, Larue T. (1995), Trust: The Connecting between Organizational Theory and Philosophical Ethics, Academy of Management Review, 20(2), USA, Academy of Management, pp. 379403.

Hu, Li-tze; Bentler, P. M. y Kano, Yukata (1992), Can Test Statistics in Covariance Structure Analysis be Trusted, Psychological Bulletin, 112, USA, American Psychological Association, pp. 351-362.

Jap, Sandy D. (1999), Pie-expansion Efforts: Collaboration Processes in Buyer-supplier Relationships, Journal of Marketing Research, 36(4), USA, American Marketing Association, pp. 461-475.

Jöreskog, Karl G. y Sörbom, Dag (1993), LISREL 8: Structural Equation Modeling with the SIMPLIS Command Languaje. Scientific Software International, Chicago-Illinois.

Kumar, Nirmalya; Hibbard, Jonathan.D. y Stern, Louis W. (1994), The nature and consequences of marketing 
channel intermediary commitment, Cambridge, MA: Marketing Science Institute.

Kumar, Nirmalya; Scherr, Lisa K. y Steenkamp, Jan B. (1995), The Effects of Perceived Interdependence on Dealer Attitudes, Journal of Marketing Research, 32(3), USA, American Marketing Association, pp. 348-56.

Li, Dahui; Browne, Glenn B. y Chau, Patrick Y.K. (2006), An Empirical Investigation of Web Site Use Using Commitment-Based Model, Decision Science, 37(3), UK, Blackwell, pp. 427-444.

Luque, Teodoro. (2000), Técnicas de Análisis de Datos en Investigación de Mercados. Pirámide, Madrid.

Macintosh, Gerrard; Anglin, Kenneth A.; Szymanski, David M. y Gentry, James W. (1992), Relationship Development in Selling: a Cognitive Analysis, Journal of Personal Selling \& Sales Management, 12(4), USA, PSENEF, pp. 23-34

Mayer, Roger C.; Davis, James H. y Shoorman, F. David (1995), An Integrative Model of Organizational Trust, Academy of Management Review, 20, USA, Academy of Management pp. 709-734.

Meyer, John P.; Allen, Natalie J. y Smith, Catherine A. (1993), Commitment to Organizations and Occupations: Extension and Test of a Three-Component Conceptualization, Journal of Applied Psychology, 78(4), USA, American Psychological Association, pp. 538-551.

Meyer, John P. y Herscovitch, Lynne (2001), Commitment in the Workplace: Toward a General Model, Human Resource Management Review, 1(3), USA, Elsevier, pp. 299-326.

Moorman, Christine; Deshpande, Rohit y Zaltman, Gerald (1993), Factors Affecting Trust in Market Research
Relationship, Journal of Marketing, 57(1), USA, American Marketing Association, pp. 81-101.

Moorman, Christine; Zaltman, Gerald y Deshpande, Rohit (1992), Relationships between Providers and Users of Market Research: The Dynamics of Trust within and between Organizations, Journal of Marketing Research, 29, USA, American Marketing Association, pp. 314-29.

Morgan, Robert M. y Hunt, Shelby D. (1994), The Commitment-Trust Theory of Relationship Marketing, Journal of Marketing, 58(2), USA, American Marketing Associaction, pp. 20-38.

Mukherjee, Avinandan y Nath, Prithwiraj (2003), A Model of Trust in Online Relationship Banking, International Journal of Bank Marketing, 21(1), UK, Emerald Group, pp. 5-15.

N'Goala, Gilles. (2007), Customer Switching Resistance (CSR): The Effects of Perceived Equity, Trust and Relationship Commitment, International Journal of Service Industry Management, 18(5), UK, Emerald Group, pp. 510533.

Norusis, Marija J. (1993), SPSS. Statistical Data Analysis. SPSS Inc.

Nunnally, Jum C. (1978), Psychometric Theory. 2nd edition, McGraw-Hill, New York.

O'Reilly, Charles A. y Chatman, Jennifer A. (1986), Organizational Commitment and Psychological Attachment: The Effects of Compliance, Identification, Internalization on Pro-Social Behaviors, Journal of Applied Psychology, 71, USA, American Psychological Association, pp. 492-99

Perry, Monica L.; Sengupta, Sanjit y Krapfel, Robert (2004), Effectiveness of Horizontal Strategic Alliances in Technologically Uncertain Environments: Are Trust and Commitment Enough?, 
Journal of Business Research, 57 , USA, Elsevier, pp. 951-956.

Pritchard, Mark P.; Havitz, Mark E. y Howard, Dennis R. (1999), Analyzing the Commitment-Loyalty Link in Service Context, Journal of the Academy of Marketing Science, 27(3), USA, Academy of Marketing Science, pp. 333348.

Rexha, Nexhmi; John, Russel P. y Shang, Audrey S. (2003): The Impact of the Relational Plan on Adoption of Electric Banking, Journal of Services Marketing, 17(1), UK, Emerald Group, pp. 53-67.

Roberts, Keith; Varki, Sajeev y Brodie, Rod (2003), Measuring the Quality of Relationships in Consumer Services: An Empirical Study, European Journal of Marketing, 37(1/2), UK, Emerald Group, pp. 169-196.

Sharma, Neeru y Patterson, Paul G. (1999), The Impact of Communication Effectiveness and Service Quality on Relationship Commitment in Consumer Professional Services, Journal of Services Marketing, 13, UK, Emerald Group, pp. 151-170

Sharma, Neeru y Patterson, Paul G. (2000), Switching Costs, Alternative Attractiveness and Experience as Moderators of Relationship Commitment in Professional, Consumer Services, International Journal of Service Industry Management, 11, UK, Emerald Group, pp. 470-490.

Sharma, Neeru; Young, Louise y Wilkinson, Ian (2006), The Commitment Mix: Dimensions of Commitment in International Trading Relationships in India, Journal of International Marketing 14(3), USA, American Marketing Association, pp. 64-91.

Siguaw, Judy A.; Simpson, Penny M. y Baker, Thomas L. (1998), Effects of Supplier Market Orientation on Distributor Mar- ket Orientation and the Channel Relationship: The Distributor Perspective, Journal of Marketing, 62, USA, American Marketing Association, pp. 99-111.

Sirdeshmukh, Deepak; Singh, Jagdip y Sabol, Barry (2002), Consumer Trust, Value, and Loyalty in Relational Exchanges, Journal of Marketing, 66, USA, American Marketing Association, pp. 15-37.

Smith, J. Brock y Barclay, Donald W. (1997), The Effects of Organizational Differences and Trust on the Effectiveness of Selling Partner Relationships, Journal of Marketing, 61(1), USA, American Marketing Association, pp. 3-21.

Stern, Barbara B. (1997), Advertising Intimacy: Relationship Marketing and the Services Consumer, Journal of Advertising, 4, USA, M.E. Sharp Inc., pp. 7-19.

Suh, Bomil y Han, Ingoo (2003), Effect of Trust on Customer Acceptance of Internet Banking, Journal of Electronic Commerce Research and Applications. 1, USA, Elsevier, pp. 247-263.

Suganthi, B.; Balachandher, K.G. y Balachandran, G. (2001), Internet Banking Patronage: An Empirical Investigation of Malaysia, Journal of Internet Banking and Commerce, disponible en: www. arraydev.com/commerce/jibc/0103_01.htm.

Tan, Soo J. (1999), Strategies for Reducing Customer's Risk Aversion and Internet Shopping, Journal of Consumer Marketing, 16, UK, Emerald Group, pp. 163-180.

Tax, Stephen S.; Brown, Stephen W. y Chandrashekaran, Murali (1998), Customer Evaluations of Service Complaint Experiences: Implications for Relationship Marketing, Journal of Marketing, 62(2), USA, American Marketing Association, pp. 60-76. 
Tellefsen, Thomas y Thomas, Gloria P. (2005), The Antecedents and Consequences of Organizational and Personal Commitment in Business Service Relationships, Industrial Marketing Management, 34 (1), USA, Elsevier, pp. 23-37.

Wagner, Janet y Rydstrom, Gabrielle (2001), Satisfaction, Trust and Commitment in Consumers Relationships with Online Retailers, European Advances in Consumer Research, 5, USA, Association for Consumer Research, pp. 276-281.

Walter, Achim y Ritter, Thomas (2003), The Influence of Adaptations, Trust, and Commitment on Value-Creating Functions of Customer Relationships, Journal of Business \& Industrial Marketing, 18(4/5), UK, Emerald Group, pp. 353-365.
Wetzels, Martin; de Ruyter, Ko y Lemmink, Jos (2000), Antecedents and Consequences of Service Quality in Business-toBusiness Services, en: Handbook of Services Marketing and Management, (Eds.) Swartz, T.A. y lacobucci, D. Thousand Oaks, CA: Sage, pp.343-356.

Wilson, David T. (1995), An Integrated Model of Buyer-Seller Relationships, Journal of the Academy of Marketing Science, 23, USA, Academy of Marketing Science, pp. 335-45.

Young, Louise y Denize, Sara (1995), A Concept of Commitment: Alternative Views of Relational Continuity in Business Service Relationships, Journal of Business \& Industrial Marketing, 10(5), UK, Emerald Group, pp. 22-37. 